\title{
Asynchronous behavior of double-quiescent elementary cellular automata
}

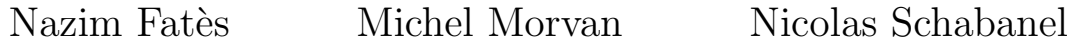 \\ Éric Thierry \\ LIP (UMR CNRS, ÉNS Lyon, INRIA, Univ. Claude Bernard Lyon I) \\ 46 allée d'Italie, 69364 Lyon Cedex 07, France.
}

\begin{abstract}
In this paper we propose a probabilistic analysis of the asynchronous behavior of elementary finite cellular automata (i.e. $\{0,1\}$ states, radius 1 and unidimensional) for which both states are quiescent (i.e. $(0,0,0) \mapsto 0$ and $(1,1,1) \mapsto 1)$. It has been experimentally shown in previous works that introducing asynchronism in the global function of a cellular automata was perturbing its behavior, but as far as we know, only few theoretical work exists on the subject. The cellular automata we consider live on a ring of size $n$ and asynchronism is introduced as follow: at each time step one cell is selected uniformly at random and the transition is made on this cell while the others stay in the same state. Under these conditions, we prove that the considered cellular automata can be classified relatively to their expected convergence time: Among the sixty-four cellular automata belonging to the class we consider, we show that nine of them diverge on all non-trivial configurations while the fifty five other always converge to a fixed point. We then study the convergence time of these fifty five automata and show that it can only take the following values: either $0, \Theta(n \ln n), \Theta\left(n^{2}\right), \Theta\left(n^{3}\right)$, or $\Omega\left(n 2^{n}\right)$. More than that, we prove that the global behavior of any of these cellular automata is fully determined by reading its code. We end the paper by showing how some of these results can be extended to another kind of asynchronism in which at each time step, each cell has independently the same fixed probability to make its transition.
\end{abstract}

\section{Introduction}

The aim of this article is to analyze theoretically the asynchronous behavior of unbounded finite cellular automata. During the last two decades, several empirical studies $[3,11,8,1,12,4]$ have shown that certain cellular automata behavior change drastically under asynchronous behavior. In particular, $[1,5]$ observe that finite size Game of Life space-time diagrams under synchronous and asynchronous updating differ qualitatively. For instance, fixed size Game of Life exhibits convergence to cycles of arbitrary length under synchronous updating, while appears to converge towards a fixed point under asynchronous dynamics [1].

Originally cellular automata were introduced by Von Neumann [13] to model real world. They still are widely used to model systems involving a huge number of interacting elements such as agents in economy, particles in physics, proteins in biology, etc. In most of these applications, in particular in many real system models, agents are not synchronous. Interestingly enough, in spite of this latent lack of synchronism, real living systems are very resilient over time. One might then expect the cellular automata used to model these systems to be robust to asynchronism and other kind of failure as well (such as misreading the state of the neighbors). Surprising enough, it turns out that the resilience to asynchronism varies widely from one automata to another (e.g., $[1,4]$ ). In particular, the asynchronous aspect of space-time diagrams of cellular automata may differ radically from their synchronous ones. 
As far as we know, the question of the importance of perfect synchrony on the behavior of a cellular automaton is not yet understood theoretically. To our knowledge, only Gács shows in [6] undecidability results on invariance with respect to the update history. Studies have also been led in the more general context of probabilistic cellular automata regarding the question of the existence of stationary distribution on infinite configurations (see [9] for a state of the art).

In this paper, we are able to quantify the convergence time and describe the shape of the space-time diagrams for a large class of cellular automata under a certain type of asynchronous updating. We focus on double-quiescent elementary automata. We show that among these sixty four automata, nine diverge on all non-trivial configurations (see Theorem 15), and the fifty five other always converge to a fixed point (see Theorem 1). The study of the convergence time of these fifty five automata on (spatially) periodic configurations, shows that it can only take the following values: either $0, \Theta(n \ln n), \Theta\left(n^{2}\right), \Theta\left(n^{3}\right)$, or $\Omega\left(n 2^{n}\right)$, where $n$ is the size of the configurations, i.e., the size of the ring. One of the most striking result is that the asynchronous global behavior of double quiescent elementary automata is obtained simply by reading the code of their local transition rules (see Tab. 1), which is known to be a difficult problem in general. Furthermore, the asynchronous behavior of all automata is characterized by this convergence time: all automata with the same convergence time present the same kind of space-time diagrams. Remark that the asynchronous behavior of some very simple automata (like the Shift) actually contains intricate stochastic processes that are currently under investigation in mathematics and physics: e.g., the asynchronous behavior of the shift automaton is intimately related to annihilating random walks, studied for instance in [10]. Our results rely on coupling the automata with a proper random process for each class.

Definitions and our main result are given in Section 2. Section 3 presents basic properties of DQECAs that will be used in the following sections. Section 4 is a technical section that develops probabilistic tools that will be used to analyze the automata. Section 5 analyzes in details the asynchronous behavior of each automaton. Some of our results extend to other type of asynchronism and are discussed in the last section of the paper.

\section{Definition, Notations and Main Results}

In this paper, we consider two-state cellular automata on finite size configurations.

Definition 1 An Elementary Cellular Automata (ECA) is given by its transition function $\delta$ : $\{0,1\}^{3} \rightarrow\{0,1\}$. We denote by $Q=\{0,1\}$ the set of states. A state $q$ is quiescent if $\delta(q, q, q)=q$. An ECA is double-quiescent (DQECA) if states 0 and 1 are quiescent.

$A$ finite configuration with periodic boundary conditions $x \in Q^{\mathbb{Z} / n \mathbb{Z}}$ is a word indexed by $\mathbb{Z} / n \mathbb{Z}$ with letters in $Q$. We denote by $U=\mathbb{Z} / n \mathbb{Z}$ the set of cells. For a given pattern $w \in Q^{*}$, we denote by $|x|_{w}=\#\left\{i: x_{i+1} \ldots x_{i+|w|}=w\right\}$ the number of occurrences of $w$ in configuration $x$.

We consider two kinds of dynamics for ECA: the classic synchronous dynamics and the stepdriven asynchronous dynamics.

Definition 2 (Synchronous Dynamics) The synchronous transition function $S_{\delta}: Q^{U} \rightarrow Q^{U}$ of an ECA $\delta$, associates to each configuration $x$ the configuration $y$, such that for all $i$ in $U$, $y_{i}=\delta\left(x_{i-1}, x_{i}, x_{i+1}\right)$.

The synchronous dynamics is the classic dynamics of cellular automata, where the transition function is applied at each (discrete) time step to each cell simultaneously. We consider here 
asynchronous dynamics where at each time step the transition function is applied to a subset of cells. There are many different ways to define such asynchronous updating of the cells of a cellular automata. The first quantitative study of the influence of the way cells are updated in CA, were carried out by Schönfisch and de Roos [12]. The authors use explicit functions for updating the cells and show that the evolution of a cellular automaton might strongly depend on the correlation between the spatial arrangement of cells and the order of their updates. (For example, if the cells are arranged in a line, one could consider the possibility of updating the cells one-by-one from left to right.) The correlation between the updating order and the spatial position of the cells is analytically estimated and it appears that for some type of updating methods, the evolution of the cellular automaton becomes strongly dependent on the lattice size. The important result is that among the different update methods studied, the only method which did not introduce any spurious correlations consisted in selecting, at every time step, each cell of the lattice independently with a given probability; we call this kind of asynchronism Bernouilli asynchronism. In the present work, we mainly consider a little variation of this particular type of asynchronism, called step-driven asynchronous dynamics in which we add the constraint that only one cell is randomly chosen at each time step. In some way, this can be seen as the most asynchronous possible regime and could correspond for example to an updating process with continuous time where cells are updated independantly at random dates. At the end of the paper, we show how our result extend to Bernouilli asynchronism.

Definition 3 (Step-driven Asynchronous Dynamics) The step-driven asynchronous transition function $A S_{\delta}: Q^{U} \rightarrow Q^{U}$ of an $E C A \delta$, associates to a configuration $x$, a random configuration $y$, such that $y_{j}=x_{j}$ for $j \neq i$, and $y_{i}=\delta\left(x_{i-1}, x_{i}, x_{i+1}\right)$, where $i$ is uniformly chosen at random in $U . A S_{\delta}$ could equivalently be seen as a function with two arguments, the configuration $x$ and the random index $i \in U$. For a given $E C A \delta$, we denote by $x^{t}$ the random variable for the configuration obtained by $t$ applications of the asynchronous transition function $A S_{\delta}$ on configuration $x$, i.e., $x^{t}=\left(A S_{\delta}\right)^{t}(x)$.

Definition 4 (Fixed point) We say that a configuration $x$ is a fixed point for $\delta$ for the asynchronous dynamics if $A S_{\delta}(x)=x$ whatever the choice of the cell to update is. $\mathcal{F}_{\delta}$ denotes the set of fixed points for $\delta$.

The set of fixed points of the asynchronous dynamics is clearly identical to $\left\{x: S_{\delta}(x)=x\right\}$ the set of fixed points of the synchronous dynamics. Note that every DQECA admits two trival fixed points, $0^{U}$ and $1^{U}$.

Definition 5 (Worst Expected Convergence Time) Given an ECA $\delta$ and a configuration $x$, we denote by $T_{\delta}(x)$ the random variable for the time to reach a fixed point from configuration $x$ under step-driven asynchronous dynamics, i.e., $T_{\delta}(x)=\min \left\{t: x^{t} \in \mathcal{F}_{\delta}\right\}$. The worst expected convergence time $T_{\delta}$ of ECA $\delta$ is :

$$
T_{\delta}=\max _{x \in Q^{U}} \mathbb{E}\left[T_{\delta}(x)\right]
$$

We can now state our main theorem.

Theorem 1 (Main result) The 64 DQECAs can be classified according to their asynchronous behavior into 6 categories. The worst expected convergence time under sequential updating of a given $D Q E C A$ is either $0, \Theta(n \ln n), \Theta\left(n^{2}\right), \Theta\left(n^{3}\right), \Omega\left(n 2^{n}\right)$ or the DQECA diverges on any configuration that is neither $0^{U}$ nor $1^{U}$ nor, when $n$ is even, $(01)^{n / 2}$. 


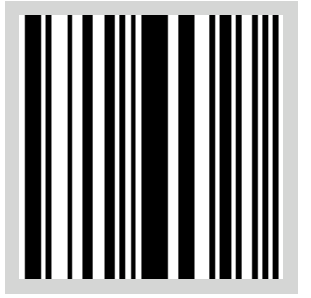

(a) $\varnothing 204$
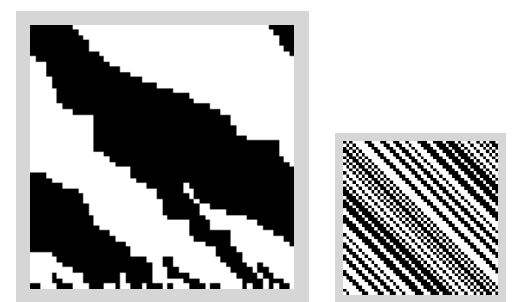

(d) BDEG 170

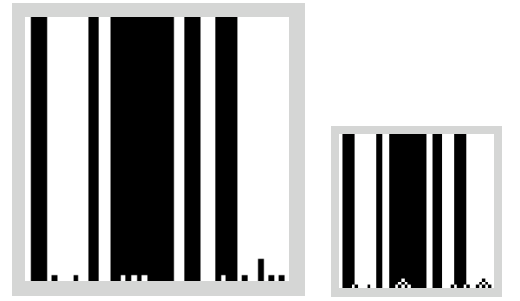

(b) DE 232

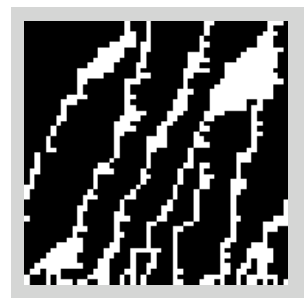

(e) BCEF 210
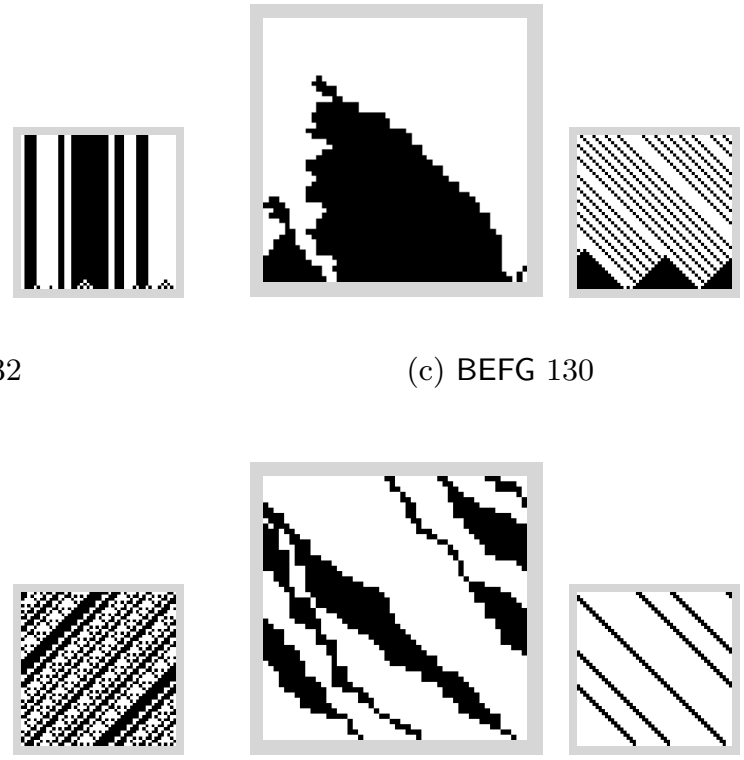

(c) BEFG 130

(f) BG 142

Figure 1: Examples of space-time diagrams under asynchronous and synchronous dynamics for each type of convergence, with $n=50$. For each automaton, the larger left and the smaller right diagrams are respectively examples of asynchronous and synchronous dynamics. White and black pixels respectively stand for states 0 and 1. The $k$-th line from bottom is the configuration at time $t=50 k$ for the asynchronous dynamics, and at time $t=k$ for the synchronous one. Note that automata (a), (b) and (d) are respectively the classic Identity, Majority and Shift rules. Each automata is described by two codes: a number, which is the classic Wolfram's number, and a sequence of letters, which will be introduced later in the paper.

Furthermore, the exact behavior of the different DQECAs is the same within each class, and is obtained by simply reading its code. This is illustrated in Tab. 1, which will be explained in the following section.

Figure 2 gives examples of the asynchronous space-time diagrams of a representative of each class. It is interesting to notice that except for the two first classes, they considerably differ from the corresponding synchronous space-time diagrams (in small).

\section{Basic properties of DQECAs}

The transition function $\delta$ of an ECA is given by the set of its eight transitions $\delta(000), \ldots, \delta(111)$,

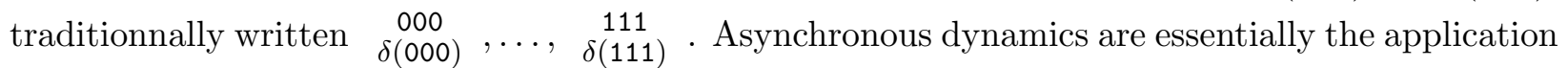
of a kind of product of Identity with the transition function. This motivates the following code that describes each ECA by its differences to the Identity automaton. We use this notation rather than the classic Wolfram's one [14] since in the latter it is not immediate to infer the local behavior of the cellular automaton just by looking at its code. However, in order to allow comparison with other work we still indicate the classic Wolfram number in Tab. 1.

Notation 1 A transition is active if it changes the state of the cell where it is applied. We label each active transition by a letter as follow: 


\begin{tabular}{|c|c|c|c|c|c|c|c|}
\hline Behavior & ECA & Rule & 01 & 10 & 010 & 101 & $\begin{array}{l}\text { Worst expected con- } \\
\text { vergence time }\end{array}$ \\
\hline Identity & $204 \quad(1)$ & $\varnothing$ & $\cdot$ & $\cdot$ & . & . & 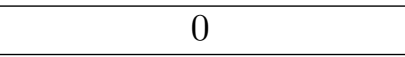 \\
\hline \multirow{2}{*}{ Coupon collector } & $200 \quad(2)$ & $\mathrm{E}$ &. &. & + & $\cdot$ & \multirow{2}{*}{$\Theta(n \ln n)$} \\
\hline & $232 \quad(1)$ & $\mathrm{DE}$ & $\cdot$ & $\cdot$ & + & + & \\
\hline \multirow{8}{*}{ Monotonic } & $206 \quad(4)$ & $\bar{B}$ & $\leftarrow$ & 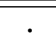 & $\overline{.}$ & $\overline{.}$ & \multirow{10}{*}{$\Theta\left(n^{2}\right)$} \\
\hline & $132 \quad(2)$ & $\mathrm{BC}$ & $\leftarrow$ & $\rightarrow$ & . & . & \\
\hline & $234 \quad(4)$ & BDE & $\leftarrow$ & $\cdot$ & + & + & \\
\hline & $160 \quad(2)$ & $\mathrm{BCDE}$ & $\leftarrow$ & $\rightarrow$ & + & + & \\
\hline & $202 \quad(4)$ & $\mathrm{BE}$ & $\leftarrow$ & $\cdot$ & + & . & \\
\hline & $192 \quad(4)$ & EF & $\rightarrow$ & . & + & . & \\
\hline & $118 \quad(2)$ & BCE & $\leftarrow$ & $\rightarrow$ & + & . & \\
\hline & $128 \quad(2)$ & EFG & $\rightarrow$ & $\leftarrow$ & + & . & \\
\hline \multirow{2}{*}{ Biased Random Walk } & $242 \quad(4)$ & BCDEF & $\overleftarrow{4}$ & $\rightarrow$ & + & + & \\
\hline & $130 \quad(4)$ & BEFG & $\overleftarrow{4}$ & $\leftarrow$ & + & . & \\
\hline \multirow{6}{*}{ Random Walk } & $226 \quad(2)$ & BDEF & $\overleftarrow{厶}$ & . & + & + & \multirow{6}{*}{$\Theta\left(n^{3}\right)$} \\
\hline & $170 \quad(2)$ & BDEG & $\leftarrow$ & $\leftarrow$ & + & + & \\
\hline & $178 \quad(1)$ & BCDEFG & $\overleftarrow{\zeta}$ & $\overleftarrow{\zeta}$ & + & + & \\
\hline & $194 \quad(4)$ & BEF & $\overleftarrow{厶}$ & $\cdot$ & + & . & \\
\hline & $138 \quad(4)$ & BEG & $\leftarrow$ & $\leftarrow$ & + & . & \\
\hline & $146 \quad(2)$ & BCEFG & 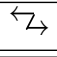 & 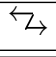 & + & . & \\
\hline Biased Random Walk & $210 \quad(4)$ & BCEF & $\overleftarrow{\zeta}$ & $\rightarrow$ & + & . & $\Omega\left(n 2^{n}\right)$ \\
\hline \multirow{4}{*}{ Divergent } & $156 \quad(2)$ & $\mathrm{BF}$ & $\overleftarrow{4}$ &. & . & . & \multirow{4}{*}{ Divergent } \\
\hline & $142 \quad(2)$ & BG & $\leftarrow$ & $\leftarrow$ & . & $\cdot$ & \\
\hline & $134 \quad(4)$ & $\mathrm{BCF}$ & $\overleftarrow{4}$ & $\rightarrow$ & $\cdot$ & . & \\
\hline & $150 \quad(1)$ & BCFG & $\overleftarrow{\zeta}$ & $\overleftarrow{4}$ & . & . & \\
\hline
\end{tabular}

Table 1: Behavior of DQECA under sequential updating

\begin{tabular}{|c|c|c|c|c|c|c|c|}
\hline A & B & C & D & E & F & G & H \\
\hline 000 & 001 & 100 & 101 & 010 & 011 & 110 & 111 \\
1 & 1 & 1 & 1 & 0 & 0 & 0 & 0 \\
\hline
\end{tabular}

Each ECA is fully determined by its active transitions. We label each ECA by the set of the corresponding letters.

Note that with these notations, DQECAs are exactly ECAs having a label not containing neither A nor H. A case study of the action of conjugation ${ }^{1}$ and reflection ${ }^{2}$, shows that among the 64 DQECAs, we shall w.l.o.g. only consider the 24 DQECAs listed in Tab. 1. For each of these 24 DQECAs, the number of the equivalent automata under reflection and conjugation is written within parentheses after their classic ECA code in the table.

From now on and until Section 6, we only consider the step-driven asynchronous dynamics (with uniform choice); this will be implicit in all the following propositions.

Our results rely on the study of the evolution of the "regions" in the space-time diagram (i.e., of the intervals of consecutive 0s or 1s in configuration $x^{t}$ ). The key observation is that for DQECAs,

${ }^{1}$ The conjugate of a configuration is obtained by exchanging 0 s and $1 \mathrm{~s}$.

${ }^{2}$ The reflection of a configuration is obtained by mirroring the configuration. 
under step-driven asynchronous dynamics, the number of regions is non-increasing since no new region can be created; furthermore, only regions of length one can disappear (see Fig. 2). We denote by $Z(x)=|x|_{01}\left(=|x|_{10}\right)$ the number of alternations from 0 to 1 in configuration $x$, which will be our counter for the number of regions.

Proposition 2 For any DQECA, $Z\left(x^{t}\right)$ is a non-increasing function of time. Furthermore, $Z\left(x^{t+1}\right)<Z\left(x^{t}\right)$ if and only if $x^{t+1}$ is obtained from $x^{t}$ by applying a transition $\mathrm{D}$ or $\mathrm{E}$ at time $t$, and then $Z\left(x^{t+1}\right)=Z\left(x^{t}\right)-1$.

Proof. Assume that $x^{t+1} \neq x^{t}$. Consider that the transition function is applied on cell $i$ at time $t$, i.e., $x_{j}^{t+1}=x_{j}^{t}$ for $j \neq i$ and $x_{i}^{t+1}=\delta\left(x_{i-1}^{t}, x_{i}^{t}, x_{i+1}^{t}\right) \neq x_{i}^{t}$. If $x_{i-1}^{t} \neq x_{i+1}^{t}$, then the number of patterns 01 or 10 can not change whatever $x_{i}^{t+1}$ and $x_{i}^{t}$ are, i.e., $Z\left(x^{t+1}\right)=Z\left(x^{t}\right)$. Otherwise, $x_{i-1}^{t}=x_{i+1}^{t}$. Since both states 0 and 1 are quiescent, if $x_{i-1}^{t}=x_{i}^{t}=x_{i+1}^{t}$, then $x^{t+1}=x^{t}$ which contradicts our hypothesis. Thus, $x_{i}^{t+1}=x_{i-1}^{t}=x_{i+1}^{t} \neq x_{i}^{t}$ and $Z\left(x^{t+1}\right)=Z\left(x^{t}\right)-1$. Note that the last case corresponds exactly to the application of transition $\mathrm{D}$ or $\mathrm{E}$.

On the one hand, transitions $\mathrm{D}$ and $\mathrm{E}$ are thus responsible for decreasing the number of regions in the space-time diagram: D "erases" the 1-regions and E the 0-regions. On the other hand, transitions $B$ and $F$ act on patterns 01. Intuitively, transition B moves a pattern 01 to the left, and transition $F$ moves it to the right. In particular, patterns 01 perform a kind of random walk for DQECA with both transitions B and F. Similarly, transitions C and G act on patterns 10. Transition $C$ moves a pattern 10 to the right, and transition $G$ moves it to the left. The arrows in Tab. 1 represents the different behavior of the patterns: $\leftarrow$ or $\rightarrow$ for left or right moves of the

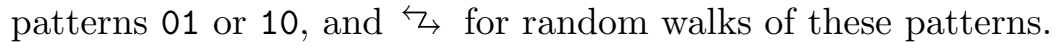

The following lemma characterizes the fixed points of a given DQECA according to its code.

Lemma 3 If a DQECA admits a fixed point $x$ that is not $0^{U}$ or $1^{U}$, then:

- if its rule contains transition $\mathrm{B}$ or $\mathrm{C}$, then all $\mathrm{O}$ s in $x$ are isolated $^{3}$;

- if its rule contains transition $\mathrm{F}$ or $\mathrm{G}$, then all $1 \mathrm{~s}$ in $x$ are isolated;

- if its rule contains transition $\mathrm{D}$, then none of the $0 s$ in $x$ is isolated;

- if its rule contains transition $\mathrm{E}$, then none of the $1 s$ in $x$ is isolated.

Proof. If the DQECA has transition $\mathrm{B}$, then one can apply this transition to any configuration $x \neq 0^{U}$ with (at least) two consecutive 0 s, and obtain a configuration $x^{\prime} \neq x$. Thus, any such configuration is not a fixed-point of such a DQECA. One can treat similarly the other cases.

The next section is a technical section that analyzes particular random walk-like processes that will be used as tools to obtain our bounds on the convergence time.

\footnotetext{
${ }^{3}$ A letter $a$ at a given location in a word $w$ is isolated if none of its two neighboring letters in $w$ is the letter $a$.
} 


\section{Probabilistic toolbox}

\subsection{Quadratic DQECA toolbox}

Let $\epsilon>0$, and two non-negative integers $m$ and $m^{\prime}$, and $\left(X_{t}\right)_{t \in \mathbb{N}}$ a sequence of random variables with values in $\left[-m, m^{\prime}\right]$, with a suitable filtration ${ }^{4}\left(\mathcal{F}_{t}\right)_{t \in \mathbb{N}}$. The following lemma bounds the convergence time of a random variable that decreases by a constant on expectation.

Lemma 4 Assume that if $X_{t}>0$, then $\mathbb{E}\left[X_{t+1}-X_{t} \mid \mathcal{F}_{t}\right] \leqslant-\epsilon$. Let $T=\min \left\{t: X_{t} \leqslant 0\right\}$ denote the random variable for the first time $t$ where $X_{t} \leqslant 0$. Then, $\mathbb{E}[T] \leqslant\left(m+\mathbb{E}\left[X_{0}\right]\right) / \epsilon$.

Proof. See Appendix, page 13.

\subsection{Cubic DQECA toolbox}

Let $\epsilon>0$ and $\left(X_{t}\right)_{t \in \mathbb{N}}$ a sequence of random variables with values in $\{0, \ldots, m\}$, with a suitable filtration $\left(\mathcal{F}_{t}\right)_{t \in \mathbb{N}}$.

Definition 6 We following two types of process will be extensively used in the next section:

- We say that $\left(X_{t}\right)_{t \in \mathbb{N}}$ is of type I if:

- $\left(X_{t}\right)$ is a martingale (i.e., for all $t, \mathbb{E}\left[X_{t+1} \mid \mathcal{F}_{t}\right]=X_{t}$ ); and

- for all $t$, if $0<X_{t}<m$, then $\operatorname{Pr}\left\{X_{t+1} \geqslant X_{t}+1 \mid \mathcal{F}_{t}\right\}=\operatorname{Pr}\left\{X_{t+1} \leqslant X_{t}-1 \mid \mathcal{F}_{t}\right\} \geqslant \epsilon$.

- We say that $\left(X_{t}\right)_{t \in \mathbb{N}}$ is of type II if:

- for all $t$, if $X_{t}<m$, then $\mathbb{E}\left[X_{t+1} \mid \mathcal{F}_{t}\right]=X_{t}$ (that is to say, $\left(X_{t}\right)$ behaves as a martingale except when $X_{t}=m$ ); and

- for all $t$, if $0<X_{t}<m$, then $\operatorname{Pr}\left\{X_{t+1} \geqslant X_{t}+1 \mid \mathcal{F}_{t}\right\}=\operatorname{Pr}\left\{X_{t+1} \leqslant X_{t}-1 \mid \mathcal{F}_{t}\right\} \geqslant \epsilon$;

- and for all $t$, if $X_{t}=m$, then $\operatorname{Pr}\left\{X_{t+1} \leqslant m-1 \mid \mathcal{F}_{t}\right\} \geqslant \epsilon$.

Note that when $\left(X_{t}\right)$ is of type I, if for some $t, X_{t} \in\{0, m\}$, then $X_{t^{\prime}}=X_{t}$ for all $t^{\prime} \geqslant t$, because $\left(X_{t}\right)$ is a martingale bounded between 0 and $m$ (i.e., $\{0, m\}$ are the fixed points of any type I sequence). When $\left(X_{t}\right)$ is of type II, if for some $t, X_{t}=0$, then $X_{t^{\prime}}=X_{t}$ for all $t^{\prime} \geqslant t$, because $\left(X_{t}\right)$ is a martingale lower bounded by 0 (i.e., 0 is the fixed point of any type II sequence).

Definition 7 The convergence time of a type I sequence $\left(X_{t}\right)$ is defined as the random variable $T=\min \left\{t: X_{t} \in\{0, m\}\right\}$. The convergence time of a type II sequence $\left(X_{t}\right)$ is similarly defined as the random variable $T=\min \left\{t: X_{t}=0\right\}$.

Lemma 5 For any type I sequence $\left(X_{t}\right)$, the expectation of $T$ under fixed $\mathcal{F}_{0}$ satisfies: $\mathbb{E}\left[T \mid \mathcal{F}_{0}\right] \leqslant X_{0}\left(m-X_{0}\right) /(2 \epsilon)$.

Lemma 6 For any type II sequence $\left(X_{t}\right)$, the expectation of $T$ under fixed $\mathcal{F}_{0}$ satisfies: $\mathbb{E}\left[T \mid \mathcal{F}_{0}\right] \leqslant X_{0}\left(2 m+1-X_{0}\right) /(2 \epsilon)$.

\footnotetext{
${ }^{4}$ A filtration $\left(\mathcal{F}_{t}\right)$ is a increasing sequence of subsets of probabilistic events, required to define properly conditional probabilities with respect to the past. Intuitively, $\mathcal{F}_{t}$ represents the set of the events that happened before time $t$. For instance, $\mathcal{F}_{t}$ may be the set of the values of $X_{0}, \ldots, X_{t}$, or in the case of a sequence built on a CA, the set of the configurations of the CA from time 0 to $t$.
} 
Proof. See Appendix, page 14.

\section{Convergence Time}

In this section, we evaluate the worst expected convergence time for each of the twenty-four representative automata in Tab. 1. Our results rely on studying the evolution of quantities computed on the random configurations $\left(x^{t}\right)$, whose convergence implies the convergence of the automaton. The upper bounds on the convergence time of these quantities are obtained by coupling them with one of the integer random process analyzed in the previous section. The lower bounds are obtained by analyzing the exact expected convergence time for a particular initial configuration (most of the time, a configuration with a single white and a single black regions).

The following functions allow us to evaluate the quantities that will be used to bound the convergence time by simply reading the label of the ECA, without having to parse the patterns in the configurations. For a given configuration $x$, we denote by $a(x), \ldots, h(x)$ the number of cells where transitions $A, \ldots, H$ may apply, i.e.:

$$
\begin{aligned}
& a(x)=|x|_{000}, \quad b(x)=|x|_{001}, \quad c(x)=|x|_{100}, \quad d(x)=|x|_{101}, \\
& e(x)=|x|_{010}, \quad f(x)=|x|_{011}, \quad g(x)=|x|_{110}, \quad h(x)=|x|_{111} \text {. }
\end{aligned}
$$

For instance, consider rule BCG. Applying the transitions A, .., D increases the number of 1s by one and applying $\mathrm{E}, \ldots, \mathrm{H}$ decreases it by one. Since, in step-driven asynchronous dynamics with uniform choice, the cell selected has probability $b(x) / n, c(x) / n, g(x) / n$ to be updated according to transition $\mathrm{B}, \mathrm{C}$ or $\mathrm{G}$ respectively, or to remain unchanged otherwise, the expected variation of the number of 1 s for configuration $x$ in one step is immediately given by $(b(x)+c(x)-g(x)) / n$.

Clearly, we have the following relationships.

Fact 7 For all configurations $x \in Q^{U}$, the following equalities hold:

$$
\begin{aligned}
|x|_{01} & =b+d=e+f=c+d=e+g=|x|_{10} \\
|x|_{001} & =b=c=|x|_{100} \\
|x|_{011} & =f=g=|x|_{110}
\end{aligned}
$$

Proof. These equations are obtained by parsing properly the string $x$.

Let us now analyse the worst expected convergence time for DQECAs. For convenience, we denote by $p=1 / n$ the probability that a given cell is updated in the asynchronous dynamics.

\section{1 "Coupon collector" DQECAs}

The behavior of the DQECAs in this class (see Fig. 1(b)) is similar to the classic Coupon Collector random process (e.g., [7]).

Theorem 8 The worst expected convergence time for DQECAs $\mathrm{E}$ and $\mathrm{DE}$ is $\Theta(n \ln n)$. The fixed points for $\mathrm{E}$ and $\mathrm{DE}$ respectively are the configurations without isolated 1 and the configurations without isolated 0 and 1. 
Proof. (Sketch) These rules simply erase either isolated 0s, isolated 1s or both. They never create any them (by Proposition 2), and reach a fixed point as soon as no more 0 or 1 are isolated (by Lemma 3). These processes are then similar to a coupon collector process that has to collect all the isolated 0s or 1s, by drawing at each time step a random location uniformly in $\{1, \ldots, n\}$. The convergence time is then bounded by $O(n \ln n)$ (see e.g., [7]). Finally, configuration $\left((010)^{\lfloor n / 3\rfloor} 0^{n} \bmod 3\right)$ provides a lower bound of $\Omega(n \ln n)$ for both rules.

\subsection{Quadratic DQECAs}

Figure 1(c) illustrates the typical space-time diagram in this class. All the results of this section are obtained by showing that a proper variant ${ }^{5}$ whose convergence implies the convergence of the DQECA, decreases by a constant on expectation.

Lemma 9 Given an initial configuration $x$, for each $D Q E C A$ B, BC, BDE, BCDE, BCDEG, BE, $\mathrm{EF}, \mathrm{BCE}, \mathrm{EFG}, \mathrm{BCEFG}$, and BEFG, there exists a sequence $\left(X_{t}\right)$ of random variable with values in $\{0, \ldots, n\}$, such that:

(a) if $X_{t}=0$, then $x^{t}$ is a fixed point.

(b) for all $t$ such that $x^{t}$ is not a fixed point, $\mathbb{E}\left[X_{t+1}-X_{t} \mid X_{t}\right] \leqslant-p$.

Proof. Consider rules B or BC. Take $X_{t}=\left|x^{t}\right|_{0}$ the number of 0 s in $x^{t}$. (a) is clear since $X_{t}=0$ implies that $x^{t}=1^{U}$. We obtain (b) by noticing that each application of transitions B or C decreases $X_{t}$ by one, and that for any non fixed-point configuration, an active transition is performed with probability $\geqslant p$. Similarly, $X_{t}=\left|x^{t}\right|_{1}$ is suitable for rules EF and EFG.

The study of the remaining rules, we need to take into account the presence of isolated 0 s and 1s. We take $X_{t}=\left|x^{t}\right|_{0}+Z\left(x^{t}\right)$ for rules BDE, BCDE, BE, BCE, and BCDEG; and $X_{t}=\left|x^{t}\right|_{1}+Z\left(x^{t}\right)$ for rule BEFG. Consider automaton BEFG. Clearly, $X_{t} \in\{0, \ldots, n\}$, and (a) $X_{t}=0$ implies that $x^{t}=0^{U}$. On the one hand, for this rule, $\mathbb{E}\left[X_{t+1}-X_{t} \mid x^{t}\right]=p \cdot(b-e-f-g)\left(x^{t}\right)-p \cdot e\left(x^{t}\right)$, since only transition $\mathrm{E}$ acts on $Z\left(x^{t}\right)$. By Fact 7 , one can rewrite $\mathbb{E}\left[X_{t+1}-X_{t} \mid x^{t}\right]=p \cdot(d+e+g)\left(x^{t}\right)$. On the other hand, if $x$ is not a fixed point, then $(b+e+f+g)(x)>0$. But by Fact 7 , if $d+e=0$, then $b=f=g$. Thus, $b+e+f+g>0$ implies $d+e+g>0$. We conclude that if $x^{t}$ is not a fixed point, we have (b). The proof is similar for all the remaining automata.

We can now state the theorem.

Theorem 10 The worst expected convergence time for DQECAs $\mathrm{B}, \mathrm{BC}, \mathrm{BDE}, \mathrm{BCDE}, \mathrm{BCDEG}$, $\mathrm{BE}, \mathrm{EF}, \mathrm{BCE}$, EFG, BCEFG, and BEFG is $\mathrm{\Theta}\left(n^{2}\right)$. Only the DQECAs $\mathrm{B}, \mathrm{BC}, \mathrm{BE}$, and $\mathrm{BCE}$ have fixed points that are distinct from $0^{U}$ and $1^{U}$, which are all the configurations where all the $0 s$ are isolated.

Proof. The property on the fixed points is a direct application of Lemma 3. Consider now one of the rules. Let $X_{t}$ be the variant given by Lemma $9 . X_{t}$ does not exactly verify the hypothesis of Lemma 4: $X_{t}$ needs to be extended beyond a fixed point if it is reached before $X_{t}=0$. We consider the random sequence $X_{t}^{\prime}$ defined as follow: $X_{t}^{\prime}=X_{t}$ if $x^{t}$ is not a fixed point, and $X_{t}^{\prime}=0$ otherwise. Thus, $X_{t}^{\prime}=0$ iff $x^{t}$ is a fixed point, and we can now apply Lemma 4 with $m=0, m^{\prime}=n$ and $\epsilon=p$ and we get $\mathbb{E}[T] \leqslant X_{0} / p=O\left(n^{2}\right)$.

\footnotetext{
${ }^{5}$ The notion of variant is also named Lyapounov function in other contexts.
} 
The lower bound $\Omega\left(n^{2}\right)$ on the convergence time is simply given by considering the following initial configuration $0^{\lceil n / 2\rceil} 1^{\lfloor n / 2\rfloor}$.

Observe that we can divide this class in two subcategories: the automata that are monotonic, for which the variant is a non-increasing function of time, and the non-monotonic, for which the variant follows a biased random walk (see Tab. 1). Interestingly enough, this distinction can be seen on the space-time diagrams.

\subsection{Cubic DQECAs}

Figure 1(d) illustrates the typical behavior of this class: one can observe that the regions in the space-time diagram follows some kind of unbiased random walk. One can observe that the frontiers between regions follow some annihilating random walks (e.g.,[10]).

All the results of this section are obtained by coupling the process with a suitable unbiased bounded random walk, such that the DQECA has converged as soon as the walk has reached any (or one distinguished) boundary.

Lemma 11 Given an initial configuration $x$, for each DQECA BDEF, BDEG, and BCDEFG, there exists an integer $m$ and a random integer sequence $\left(X_{t}\right)$ of type $I$ (see section 4.2) with values in $\{0, \ldots, m\}$, such that: for all $t$, if $X_{t}=0$ or $X_{t}=m$, then $x^{t}$ is a fixed point.

Proof. For a given random sequence $\left(X_{t}\right)_{t \in \mathbb{N}}$ we denote by $\left(\Delta X_{t}\right)_{t>0}$ the random sequence $\Delta X_{t}=$ $X_{t}-X_{t-1}$.

We take $X_{t}=\left|x^{t}\right|_{1}$ for automata BDEF, BDEG. Consider rule BDEG (the Shift 170). $X_{t}$ takes its values in $\{0, \ldots, n\}$, and $X_{t} \in\{0, m\}$ implies that $x^{t}$ is a (trivial) fixed point. Reading the code of the rule, we have for all $t, \mathbb{E}\left[\Delta X_{t+1} \mid x^{t}\right]=p \cdot(b+d-e-g)\left(x^{t}\right)=0$ by Fact 7 . $X_{t}$ is thus a martingale. For every time $t$ such that $0<X_{t}<n, \operatorname{Pr}\left\{\Delta X_{t+1} \geqslant 1 \mid x^{t}\right\}=p \cdot(b+d)\left(x^{t}\right)=p,\left|x^{t}\right|_{01}=$ $p \cdot(e+g)\left(x^{t}\right)=\operatorname{Pr}\left\{\Delta X_{t+1} \geqslant 1 \mid x^{t}\right\}$ and $\left|x^{t}\right|_{01} \geqslant 1$. $X_{t}$ is then of type II. The proof is similar for BDEG.

See Appendix, page 14 for rule BCDEFG.

Lemma 12 Given an initial configuration $x$, for each DQECA BEF, BEG, and BCEFG, there exists an integer $m$ and a random integer sequence $\left(X_{t}\right)$ of type II (see section 4.2) with values in $\{0, \ldots, m\}$, such that: for all $t$, if $X_{t}=0$, then $x^{t}$ is a fixed point.

Proof. Consider BEF. We define the process $X_{t}$ as follow. First, $X_{0}=\left|x^{0}\right|_{1}$. As long as $x^{t}$ is not a fixed point, $X_{t+1}$ depends on the neighborhood of the cell updated at time $t$. Assume that $x^{t}$ is not a fixed point. If the transition applied is $\mathrm{E}$ or $\mathrm{F}$, then $X_{t+1}=X_{t}-1$. If the transition applied is B or the neighborhood of the selected cell is 101 (i.e., the site of a fictitious transition D), then $X_{t+1}=\min \left(n-1, X_{t}+1\right)$; otherwise, $X_{t+1}=X_{t}$ (this trick is needed to make the process symmetric). Clearly, for all $t, X_{t} \in\{0, \ldots, n-1\}$ and $X_{t} \geqslant\left|x^{t}\right|_{1}$, i.e. $X_{t}$ bounds from above the number of $1 \mathrm{~s}$ in the configuration at any time $t$. As a consequence, the fixed point $0^{U}$ is reached at time $t$ if $X_{t}=0$. Assume again that $x^{t}$ is not a fixed point. If $X_{t}<n-1$, then $\mathbb{E}\left[\Delta X_{t+1} \mid x^{t}, X_{t}\right]=p \cdot(b+d-e-f)\left(x^{t}\right)=0$ by Fact 7 , and $\operatorname{Pr}\left\{\Delta X_{t+1} \geqslant 1 \mid x^{t}, X_{t}\right\}=\operatorname{Pr}\left\{\Delta X_{t+1} \leqslant\right.$ $\left.-1 \mid x^{t}, X_{t}\right\}=p \cdot(b+d)\left(x^{t}\right) \geqslant p$. Otherwise, $X_{t}=n-1$ and $\operatorname{Pr}\left\{\Delta X_{t+1} \mid x^{t}, X_{t}\right\}=p \cdot(e+f)\left(x^{t}\right) \geqslant p$. In order to get a proper process of type II we need to extend $\left(X_{t}\right)$ beyond the fixed point with two extra last steps: if $x^{t}$ is a fixed point, then $X_{t+1}=0$ or $X_{t+1}=n-1$ with respective probabilities $\frac{X_{t}}{n-1}$ and $1-\frac{X_{t}}{n-1}$; and if $X_{t+1}=n-1$, then $X_{t+2}=0$. The designed $\left(X_{t}\right)$ is then a suitable process 
of type II for rule BEF. By symmetry, exchanging $f$ and $g$, in the definition of $X_{t}$ gives a suitable process of type II for BEG.

See Appendix, page 15 for rule BCEFG.

Theorem 13 The worst expected convergence time for DQECAs BDEF, BDEG, BCDEFG, BEF, $\mathrm{BEG}$, and $\mathrm{BCEFG}$, is $\Theta\left(n^{3}\right)$. All of them admit only $0^{U}$ and $1^{U}$ as fixed point.

For DQECAs BDEF, BDEG, and BCDEFG, $O^{U}$ and $1^{U}$ can be reached from any configuration (respectively distinct from $1^{U}$ and $0^{U}$ ). For DQECAs BEF, BEG, and BCEFG, any configuration distinct from $1^{U}$ converges to $0^{U}$.

Proof. Straightforward application of Lemma 11 and 12 in combination with the probabilistic Lemmas 5 and 6 .

\subsection{Exponential DQECA}

Theorem 14 The worst expected convergence time for DQECA BCEF is $\Omega\left(n 2^{n}\right)$. Its fixed points are $0^{U}$ and $1^{U}$, and any configuration distinct from $1^{U}$ converges to $0^{U}$.

Proof. The assertion about fixed points is given by Lemma 3. The bound on the worst case expected convergence time is obtained by considering the process starting for the initial configuration $x=01^{n-1}$. The reachable configurations from $x$ are $0^{n-i} 1^{i}, 0 \leqslant i \leqslant n-1$ (up to circular permutations). The process restricted to this set of configurations is fully described by the evolution of $X_{t}=\left|x^{t}\right|_{1}$, which behaves as a biased random walk on $\{0, \ldots, n-1\}$. See Appendix, page 15 for the end of the proof.

\subsection{Diverging DQECAs}

Figure 1(f) illustrates the typical behavior of a divergent DQECA: the number of regions is conserved, and all reachable ${ }^{6}$ configurations from a given initial configuration are accessed an infinite number of times almost surely.

Theorem 15 The DQECAs BF, BG, BCF, and BCFG diverge on any configuration that is not one of the three following fixed points $0^{U}, 1^{U}$, and, if $n$ is even, $(01)^{n / 2}$. Furthermore, given an initial configuration, all reachable configurations are accessed an infinite number of times almost surely.

Proof. According to Lemma 3, the only possible non-trivial fixed points for these automata are configurations where all 0 s and all 1 s are isolated. Thus, only when $n$ is even, these automata admit an extra fixed point, $(01)^{n / 2}$, in addition to $0^{U}$ and $1^{U}$. Furthermore, according to Proposition 2, the number $Z\left(x^{t}\right)$ of alternations from 0 to 1 is constant, because none of these automata contains transitions D nor E. Thus, none of the fixed points can ever be reached from non-fixed point configurations, since $Z\left(0^{U}\right)=Z\left(1^{U}\right)=0<Z(x)<n / 2=Z\left((01)^{n / 2}\right)$ for all other configurations.

The second part of the theorem consists in proving that there exists a finite length sequence of transitions between any pair of reachable configurations (omitted).

Note that for rules BG, BCF, and BCFG the set reachable points is simply the set of configurations with the same number of alternation from 0 to 1 as the initial configuration.

\footnotetext{
${ }^{6}$ Given an initial configuration $x$, a configuration $y$ is reachable (from $x$ ) if $\operatorname{Pr}\left\{x^{t}=y\right\}>0$ for some $t$, i.e., $y$ can be obtained by a sequence of transitions on $x$.
} 


\section{Extension to other type of asynchronism}

The behavior of the DQECA under Bernouilli asynchronism is harder to analyse. However, some cases can almost directly be deduced from the previous results. So let us now assume that at each time step, each cell has a fixed probability $\alpha$ to be updated. We can then immediately state the following results on the worst expected convergence time.

E: $\Theta\left(-\frac{\ln n}{\ln (1-\alpha)}+\frac{1}{\alpha}\right)$. This comes from the fact that starting from $k$ isolated 1 s the expected number of isolated 1s after $t$ time steps is $(1-\alpha)^{t} k$.

B and BC: $\Theta\left(\frac{n}{\alpha}\right)$. At each time step before reaching a fixed point, the number of 0s decreases on average by at least $\alpha$ for B and by at least $2 \alpha$ for BC; so we can apply Lemma 4 and obtain the result.

BDEF and BDEG: $\Theta\left(\frac{n^{2}}{\alpha(1-\alpha)}\right)$. At each time step, the number of 1s remains constant on average and before reaching a fixed point the probability of increasing this number by at least one is equal to the probability to decrease it by at least one and is greater or equal to $\alpha(1-\alpha)$; so we can apply Lemma 5 and obtain the result. Let us remark that in these two cases the factor $1-\alpha$ in the denominator induces a discontinuity for $\alpha=1$. This discontinuity implies that even the introduction of a very little amount of asynchronism (by choosing $\alpha$ very closed to 1) will lead to a behavior dramatically different from the synchronous one. This can be easily seen for BDEG which is the classical shift in synchronous mode and behaves completely differently for any $\alpha$ lower than one.

BEG: $\Theta\left(\frac{n^{2}}{\alpha(1-\alpha)}\right)$. This result is obtained in two steps. First we prove it for a configuration with only one zone of 1 s by using Lemma 6 . Second, starting from a configuration with more than one zone of 1s, we couple it with another one with exactly one zone of $1 \mathrm{~s}$ by filling with $1 \mathrm{~s}$ all 0 s zones except one. We then just prove that, during the process, any 1 in the first configuration always corresponds to a 1 in the second. When the second configuration reaches $0^{U}$ (the only fixed point), so does the first one. The result follows.

\section{References}

[1] H. Bersini and V. Detours. Asynchrony induces stability in cellular automata based models. In Brooks, R. A, Maes, and Pattie, editors, Proceedings of the 4th International Workshop on the Synthesis and Simulation of Living Systems ArtificialLifeIV, pages 382-387. MIT Press, July 1994.

[2] P. Brémaud. Markov chains, Gibbs fileds, Monte Carlo simulation, and queues. Springer, 1999.

[3] R.L. Buvel and T.E. Ingerson. Structure in asynchronous cellular automata. Physica D, 1:59-68, 1984.

[4] Nazim Fatès and Michel Morvan. An experimental study of robustness to asynchronism for elementary cellular automata. Submitted, arxiv:nlin.CG/0402016, 2004.

[5] Nazim Fatès and Michel Morvan. Perturbing the topology of the game of life increases its robustness to asynchrony. In Accepted for publication in Proc. of ACRI 2004, Oct. 2004.

[6] P. Gács. Deterministic computations whose history is independent of the order of asynchronous updating. http://arXiv.org/abs/cs/0101026, 2003. 
[7] G. Grimmet and D. Stirzaker. Probability and Random Process. Oxford University Press, 3rd edition, 2001.

[8] B. A. Huberman and N. Glance. Evolutionary games and computer simulations. Proceedings of the National Academy of Sciences, USA, 90:7716-7718, August 1993.

[9] P.-Y. Louis. Automates Cellulaires Probabilistes : mesures stationnaires, mesures de Gibbs associées et ergodicité. PhD thesis, Univ. des Sciences et Technologies de Lille, Sep. 2002.

[10] M. Mattera. Annihilating random walks and perfect matchings of planar graphs. Discrete Mathematics and Theoretical Computer Science, AC:173-180, 2003.

[11] Martin A. Nowak and Robert M. May. Evolutionary games and spatial chaos. Nature (London), 359:826-829, 1992.

[12] Birgitt Schönfisch and André de Roos. Synchronous and asynchronous updating in cellular automata. BioSystems, 51:123-143, 1999.

[13] J. von Neumann. Theory of self-reproducing automata. University of Illinois Press, 1966.

[14] S. Wolfram. Universality and complexity in cellular automata. Physica D, 10:1-35, 1984.

\section{A Omitted proofs}

\section{A.1 Probabilistic Lemmas}

Proof of Lemma 4. First we prove that $\mathbb{E}[T]<\infty$ under these assumptions. For all $t<T$, we have $\epsilon \leqslant \mathbb{E}\left[X_{t}-X_{t+1} \mid \mathcal{F}_{t}\right]=\mathbb{E}\left[X_{t}-X_{t+1} \mid\left(X_{t}-X_{t+1}>\epsilon / 2\right) \wedge \mathcal{F}_{t}\right]+\mathbb{E}\left[X_{t}-X_{t+1} \mid\left(X_{t}-X_{t+1} \leqslant\right.\right.$ $\left.\epsilon / 2) \wedge \mathcal{F}_{t}\right] \leqslant\left(m+m^{\prime}\right) \operatorname{Pr}\left\{X_{t}-X_{t+1}>\epsilon / 2 \mid \mathcal{F}_{t}\right\}+\epsilon / 2$, since $\left|X_{t+1}-X_{t}\right| \leqslant m+m^{\prime}$ for all $t$. Thus $\operatorname{Pr}\left\{X_{t}-X_{t+1}>\epsilon / 2 \mid \mathcal{F}_{t}\right\} \geqslant \frac{\epsilon}{2\left(m+m^{\prime}\right)}$ for all $t<T$. This implies that from any time $t$ and any starting value $X_{t}$, we can go below 0 in $2 \mathrm{~m}^{\prime} / \epsilon$ steps with a non-negative probability independent of $\mathcal{F}_{t}$. More precisely $\operatorname{Pr}\left\{X_{t+2 m^{\prime} / \epsilon} \leqslant 0 \mid \mathcal{F}_{t}\right\} \geqslant\left(\frac{\epsilon}{2\left(m+m^{\prime}\right)}\right)^{2 m^{\prime} / \epsilon}$, which implies that the expected time to go below 0 satisfies $\mathbb{E}[T] \leqslant \frac{2 m^{\prime}}{\epsilon}+\left(\frac{2\left(m+m^{\prime}\right)}{\epsilon}\right)^{2 m^{\prime} / \epsilon}$.

Then let $Y_{t}=X_{t}+\epsilon t$. For all $t<T, \mathbb{E}\left[Y_{t+1} \mid \mathcal{F}_{t}\right] \leqslant X_{t}-\epsilon+\epsilon(t+1)=Y_{t}$. Since $T$ is almost surely finite, with finite expectation and since $\left|Y_{t+1}-Y_{t}\right| \leqslant m+m^{\prime}+\epsilon$, the Optional Stopping Theorem for the supermartingale $\left(Y_{t}\right)$ (see [7]) gives: $\mathbb{E}\left[X_{0}\right]=\mathbb{E}\left[Y_{0}\right] \geqslant \mathbb{E}\left[Y_{T}\right]=\mathbb{E}\left[X_{T}\right]+\epsilon \mathbb{E}[T]$. Thus, we have $\mathbb{E}[T] \leqslant\left(\mathbb{E}\left[X_{0}\right]-\mathbb{E}\left[X_{T}\right]\right) / \epsilon \leqslant\left(m+\mathbb{E}\left[X_{0}\right]\right) / \epsilon$.

Lemma 16 For both types of sequences, the expected convergence time is finite (i.e., $\mathbb{E}[T]<\infty$ ). Consequently, $T$ is almost surely finite (i.e. $\operatorname{Pr}\{T<\infty\}=1$ ).

Proof. The proof is similar to the beginning of Lemma 4. For a type I sequence, for all $t$, we clearly have $\operatorname{Pr}\left\{X_{t+n} \in\{0, m\} \mid \mathcal{F}_{t}\right\} \geqslant \epsilon^{n}$, which implies that the expected time to reach $\{0, m\}$ satisfies $\mathbb{E}[T] \leqslant 1 / \epsilon^{n}+n$. Replace $\{0, m\}$ by $\{0\}$ to get the same bound for type II sequences. 
Proof of Lemma 5. We suppose that all probabilities and expectations are conditioned by $\mathcal{F}_{0}$, so it will be omitted in the formulas to simplify the notation. The sequence $\left(X_{t}\right)$ is a martingale with respect to $\left(\mathcal{F}_{t}\right)$, the convergence time $T$ is clearly a stopping time with respect to $\left(\mathcal{F}_{t}\right)$ such that $\operatorname{Pr}\{T<\infty\}=1$ and $\left|X_{t}\right|$ is bounded by $m$ for all $t$. We can apply the Optional Stopping Theorem (see [7]) which gives: $\mathbb{E}\left[X_{0}\right]=\mathbb{E}\left[X_{T}\right]$. By definition of $T$ and since $X_{0}$ is constant (under fixed $\mathcal{F}_{0}$ ), we have $X_{0}=\mathbb{E}\left[X_{0}\right]=\mathbb{E}\left[X_{T}\right]=0 \operatorname{Pr}\left\{X_{T}=0\right\}+m \operatorname{Pr}\left\{X_{T}=m\right\}$. Knowing that $\operatorname{Pr}\left\{X_{T}=0\right\}+\operatorname{Pr}\left\{X_{T}=m\right\}=1$, we get: $\operatorname{Pr}\left\{X_{T}=0\right\}=1-X_{0} / m$, and $\operatorname{Pr}\left\{X_{T}=m\right\}=X_{0} / m$.

Now let $Y_{t}=X_{t}^{2}-2 \epsilon t$, the sequence $\left(Y_{t}\right)$ is a submartingale with respect to $\left(\mathcal{F}_{t}\right)$ as shown below. We denote $\Delta_{t+1}=X_{t+1}-X_{t}$ the drift at each step. Then

$$
\mathbb{E}\left[X_{t+1}^{2}-2 \epsilon(t+1) \mid \mathcal{F}_{t}\right]=X_{t}^{2}+2 X_{t} \mathbb{E}\left[\Delta_{t+1} \mid \mathcal{F}_{t}\right]+\mathbb{E}\left[\Delta_{t+1}^{2} \mid \mathcal{F}_{t}\right]-2 \epsilon-2 \epsilon t \geqslant X_{t}^{2}-2 \epsilon t,
$$

since $\mathbb{E}\left[\Delta_{t+1} \mid \mathcal{F}_{t}\right]=0$ and $\mathbb{E}\left[\Delta_{t+1}^{2} \mid \mathcal{F}_{t}\right]=\operatorname{Pr}\left\{X_{t+1} \leqslant X_{t}-1 \mid \mathcal{F}_{t}\right\}+\operatorname{Pr}\left\{X_{t+1} \geqslant X_{t}+1\right\} \geqslant 2 \epsilon$. Since $\mathbb{E}[T]<\infty$ and $\left|Y_{t+1}-Y_{t}\right| \leqslant m^{2}+2 \epsilon$, we can apply the Optional Stopping Theorem to the submartingale $Y_{t}=X_{t}^{2}-\epsilon t$ which leads to

$$
\mathbb{E}\left[X_{T}^{2}-\epsilon T\right] \geqslant \mathbb{E}\left[X_{0}^{2}\right]=X_{0}^{2}
$$

Since $\mathbb{E}\left[X_{T}^{2}-\epsilon T\right]=0^{2} \operatorname{Pr}\left\{X_{T}=0\right\}+m^{2} \operatorname{Pr}\left\{X_{T}=m\right\}-\epsilon \mathbb{E}[T]=m X_{0}-X_{0}^{2}$, it yields the final result.

Proof of Lemma 6. By definition of $T$, we have $\operatorname{Pr}\left\{X_{T}=0\right\}=1$. We now introduce the sequence $Y_{t}=X_{t}^{2}-(2 m+1) X_{t}-2 \epsilon t$ instead of $X_{t}^{2}-2 \epsilon t$. We can easily check that this sequence is a submartingale by considering the two cases: $0<X_{t}<m$ and $X_{t}=m$. We have:

$$
\begin{aligned}
\mathbb{E}\left[X_{t+1}^{2}-(2 m+1) X_{t+1}-2 \epsilon(t+1) \mid \mathcal{F}_{t}\right]= & X_{t}^{2}-(2 m+1) X_{t}-2 \epsilon t \\
& +2 X_{t} \mathbb{E}\left[\Delta_{t+1} \mid \mathcal{F}_{t}\right]+\mathbb{E}\left[\Delta_{t+1}^{2} \mid \mathcal{F}_{t}\right]-(2 m+1) \mathbb{E}\left[\Delta_{t+1} \mid \mathcal{F}_{t}\right]-2 \epsilon .
\end{aligned}
$$

If $0<X_{t}<m$, then $\mathbb{E}\left[\Delta_{t+1} \mid \mathcal{F}_{t}\right]=0$ and $\mathbb{E}\left[\Delta_{t+1}^{2} \mid \mathcal{F}_{t}\right] \geqslant 2 \epsilon$. If $X_{t}=m$, then $\mathbb{E}\left[\Delta_{t+1} \mid \mathcal{F}_{t}\right] \leqslant-\epsilon$ and $\mathbb{E}\left[\Delta_{t+1}^{2} \mid \mathcal{F}_{t}\right] \geqslant \epsilon$. In both cases,

$$
\mathbb{E}\left[X_{t+1}^{2}-(2 m+1) X_{t+1}-2 \epsilon(t+1) \mid \mathcal{F}_{t}\right] \geqslant X_{t}^{2}-(2 m+1) X_{t}-2 \epsilon .
$$

As in lemma 5, we can apply the Optional Stopping Theorem which gives

$$
\mathbb{E}\left[X_{T}^{2}-(2 m+1) X_{T}-2 \epsilon T\right] \geqslant \mathbb{E}\left[X_{0}^{2}-(2 m+1) X_{0}\right]=X_{0}^{2}-(2 m+1) X_{0} .
$$

Since $\mathbb{E}\left[X_{T}^{2}\right]=0^{2} \operatorname{Pr}\left\{X_{T}=0\right\}=0$ and $\mathbb{E}\left[X_{T}\right]=0$, we get the final result.

\section{A.2 Cubic DQECAs}

Proof of End of Lemma 11. Consider now rule BCDEFG. Because of special side effects due to transitions $\mathrm{D}$ and $\mathrm{E}$, we need to use a more intricate sequence $\left(X_{t}\right)$. We introduce two random sequences $\left(D_{t}\right)$ and $\left(E_{t}\right)$ that count respectively the number of applications of transitions $\mathrm{D}$ and $\mathrm{E}$ during time interval $[0, t)$. For $t \geqslant 0$ such that $x^{t}$ is not a fixed point, we define $X_{t}=Z\left(x^{0}\right)+$ $\left|x^{t}\right|_{1}+D_{t}-E_{t}$. Since for all $t, Z\left(x^{0}\right)-E_{t} \geqslant 0$, and $D_{t} \geqslant Z\left(x^{0}\right) \leqslant n / 2, X_{t}$ varies in $\{0, \ldots, 2 n\}$. Furthermore, if $X_{t}=0$ or $X_{t}=2 n$, then $x^{t}$ is $0^{U}$ or $1^{U}$ respectively, and the process has converged. By reading the code of the rule, $\mathbb{E}\left[\Delta X_{t+1} \mid x^{t}, D_{t}, E_{t}\right]=p \cdot(-b-c-d+e+f+g)\left(x^{t}\right)+p \cdot(e-d)\left(x^{t}\right)=0$ using Fact 7. Furthermore, assume that $x^{t}$ is not a fixed point, we have $(b+c+d+e+f+g)\left(x^{t}\right) \geqslant 1$, 
i.e., $(2 b+d+e+2 g)\left(x^{t}\right) \geqslant 1$, thus at least one of $b, d$, $e$ or $g$ is larger than 1 on $x^{t}$. We have: $\operatorname{Pr}\left\{\Delta X_{t+1} \geqslant 1 \mid x^{t}, D_{t}, E_{t}\right\}=\operatorname{Pr}\left\{\Delta X_{t+1} \leqslant-1 \mid x^{t}, D_{t}, E_{t}\right\}=p \cdot(2 e+f+g)\left(x^{t}\right)=p \cdot(b+d+e+g)\left(x^{t}\right) \geqslant$ $p$. In order to get a true process of type II, we need to extend $X_{t}$ beyond the fixed point, until it reaches either 0 or $2 n$. We proceed as follow: for $t>0$ such that $x^{t}$ is a fixed point and $X_{t} \notin\{0,2 n\}$, $X_{t+1}$ is $X_{t}+1$ or $X_{t}-1$ with equal probability $\frac{1}{2}$. $X_{t}$ is then a suitable process of type II for rule BDEFG.

Proof of End of Lemma 12. Consider finally rule BCEFG. We need a more involved process $\left(X_{t}\right)$. We define $X_{0}=\left|x^{0}\right|_{1}+Z\left(x^{0}\right)$. Assume that $x^{t}$ is not a fixed point, then the value of $X_{t+1}$ depends again on the neighborhood of the cell selected at time $t$. We denote by $\ell$ the transition corresponding to the neighborhood of the cell updated in $x^{t}$ at time $t . X_{t+1}$ is given by:

- If $X_{t} \leqslant n-2$, then: $X_{t+1}$ is $X_{t}+2$ if $\ell=\mathrm{D}$, or $X_{t}+1$ if $\ell \in\{\mathrm{B}, \mathrm{C}\}$, or $X_{t}-1$ if $\ell \in\{\mathrm{F}, \mathrm{G}\}$, or $X_{t}-2$ if $\ell=\mathrm{E}$, or $X_{t}$ otherwise.

- If $X_{t}=n-1$, then: $X_{t+1}$ is $X_{t}+1$ if $\ell \in\{\mathrm{B}, \mathrm{D}\}$, or $X_{t}-1$ if $\ell \in\{\mathrm{F}, \mathrm{E}\}$, or $X_{t}$ otherwise.

- If $X_{t}=n$, then: $X_{t+1}$ is $X_{t}-1$ if $\ell \in\{\mathrm{F}, \mathrm{G}, \mathrm{E}\}$, or $X_{t}$ otherwise.

By induction, for any $t$, we have $0 \leqslant\left|x^{t}\right|_{1}+Z\left(x^{t}\right) \leqslant X_{t} \leqslant n$, and then if $X_{t}=0$, the process has reached the fixed point $0^{U}$. Assume again that $x^{t}$ is not a fixed point, then $b+c+e+f+g \geqslant 1$, which implies that $b+e+f \geqslant 1$.

- If $X_{t} \leqslant n-2$, then: $\mathbb{E}\left[X_{t+1} \mid x^{t}, X_{t}\right]=p \cdot(b+c+2 d-2 e-f-g)\left(x^{t}\right)=0$, and $\operatorname{Pr}\left\{\Delta X_{t+1} \geqslant\right.$ $\left.1 \mid x^{t}, X_{t}\right\}=\operatorname{Pr}\left\{\Delta X_{t+1} \leqslant-1 \mid x^{t}, X_{t}\right\}=p \cdot(2 e+f+g)\left(x^{t}\right)=p \cdot(e+f+b+d)\left(x^{t}\right) \geqslant p$, because we have $b+e+f \geqslant 1$.

- If $X_{t}=n-1$, then: $\mathbb{E}\left[X_{t+1} \mid x^{t}, X_{t}\right]=p \cdot(b+d-e-f)\left(x^{t}\right)=0$, and $\operatorname{Pr}\left\{\Delta X_{t+1} \geqslant 1 \mid x^{t}, X_{t}\right\}=$ $\operatorname{Pr}\left\{\Delta X_{t+1} \leqslant-1 \mid x^{t}, X_{t}\right\}=p \cdot(b+d)\left(x^{t}\right) \geqslant p$, since we have $b+d=e+f$ and $b+e+f \geqslant 1$.

- If $X_{t}=n$, then: $\operatorname{Pr}\left\{\Delta X_{t+1} \leqslant-1 \mid x^{t}, X_{t}\right\}=p \cdot(f+g+e)\left(x^{t}\right) \geqslant p$, because we have $b+d=e+f$ and $b+e+f \geqslant 1$.

We use then the same technique as before to $\left(X_{t}\right)$ to a process of type II beyond the fixed point. The resulting sequence is then a suitable process of type II for BECFG.

\section{A.3 Exponential DQECA}

Proof of End of Theorem 14. More precisely, we have $X_{0}=n-1$, and for all $t$, if $0 \leqslant$ $X_{t} \leqslant n-2$, then $X_{t+1}=X_{t}+1$ with probability $(2 p),=X_{t}$ with probability $(1-3 p),=X_{t}-1$ with probability $(p)$, and if $X_{t}=n-1$, then $X_{t+1}=X_{t}$ with probability $(1-p),=X_{t}-1$ with probability $(p)$. The state 0 of the random walk is a fixed point and the expected convergence time $T$ for the DQECA is defined by $T=\min \left\{t: X_{t}=0\right\}$. Let $T_{i}=\mathbb{E}\left[T \mid X_{0}=i\right]$ be the expected convergence time starting from configuration $0^{n-i} 1^{i}$. First-step analysis (see [2]) gives the equations: $T_{i}=p T_{i-1}+(1-3 p) T_{i}+2 p T_{i+1}+1$ for $i \in\{1, \ldots, n-2\}, T_{n-1}=p T_{n-2}+(1-p) T_{n-1}+1$ and $T_{0}=0$. It can be checked that the solution of these equations is $T_{i}=\frac{2^{n}}{p}\left(1-2^{-i}\right)-\frac{i}{p}$ for all $i \in\{0, \ldots, n-1\}$. It implies that, when starting from the configuration $x=01^{n-1}$, the expected convergence time $T_{n-1}$ is $\Omega\left(n 2^{n}\right)$. 